\title{
Research and Implementation of Ethnic Education Resources Database Based on Ontology
}

\author{
Jun Ma \\ School of Educational Science and Technology, Northwest University for Nationalities \\ Lanzhou 730124, P.R. China \\ 364221500@qq.com
}

Keywords: Education resources; Ethnic groups; Ontology; Protégé

\begin{abstract}
The article introduced the methods of constructing education resources database for ethnic groups with Ontology taking the construction of education resources for Dongxiang ethnic group as an example in order to realize the development and sharing of education resources in ethnic areas. Meanwhile, it also discussed some related concepts, attributes and hierarchical relationship. Protégé 5.0 is used for realizing the Ontology.
\end{abstract}

\section{Introduction}

Gansu province is located in the northwest of China. The total population is 2763,65 million. It is a multi-ethnic region. There are 54 ethnic groups in Gansu. Dongxiang, Baoan, Yu-Gu are three special ethnic groups have resided in Gansu. The ethnic areas of Gansu are rich in ethnic cultural resources. It can improve the development of education in ethnic areas by constructing, preserving, inheriting and utilizing rich ethnic education resources with modern IT and advanced educational concepts.

After comparing a variety of tools and data storage models, the article will take the methods based on Ontology [1] to construct and implement ethnic education resources database. Ontology is a kind of method for knowledge representation and an effective way for knowledge sharing [2]. The aim of Ontology is to capture knowledge from related fields in order to understand the knowledge in our own field, and to determine common vocabulary and to define the relationship between them from different levels [3]. The article takes Dongxiang ethnic group as an example, and elaborates how to construct and realize the Ontology construction of its education resources; how to achieve the concept, to divide the hierarchical structure, to determine concepts and attributes and relations, to construct the examples of its education resources. The article also realizes the Ontology with Protégé.

\section{The Construction Idea of Ethnic Education Resources}

Ontology knowledge database of ethnic education resources is based on representing, organizing and storing of knowledge standard and systematically with the technology of Ontology, and it is the semantic description of education resources by means of the understandable formalized representation of machines. Firstly, the database includes the important concepts in the field of ethnic education resources; secondly, it defines the hierarchical structure, describes the attributes and relationship between the attributes, and declares the axiom in the field of knowledge in order to make computer to understand ethnic knowledge, and to reason and judge knowledge [4].

Ontology knowledge database of ethnic education resources should be suitable of teaching and learning. It can send semantic knowledge and help students to learn ethnic knowledge according to students' models. The construction steps of this ontology database based on software engineering methods as follows. 
Planning and Designing the Ontology. This stage needs to identify the meaning of constructing this database, and includes general requirements analysis and design.

Constructing the Ontology Framework. We construct the framework in seven steps after comparing all kind of modeling approach with IEEE 1074-2006 standard.

Formalizing the Ontology. It is the process of computer coding to describe the Ontology with computer language.

Evaluating and Modifying the Ontology. To analyze the correctness, consistency, scalability and validity of the ontology; to evaluate the clarity and integrity of concepts and their relations in the ontology; and to modify the ontology according to the analysis and evaluation.

Evolving the Ontology. The construction of ontology is a process of accumulation. Evolution is the nature of iteration. The ontology will be improved after every iteration. The above process is the method for constructing the ontology knowledge database of ethnic education resources according to the general principles of modeling and the characteristics of objects. The process shows as Fig. 1.

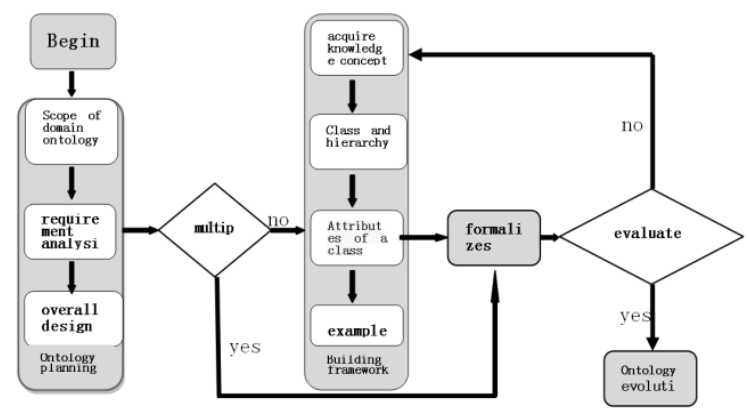

Figure 1. The process of constructing the ontology database of ethnic education resources

\section{The Realization of the Ontology of Dongxiang Ethnic Education Resources}

Constructing the ontology of Dongxiang ethnic education resources follows the process in Fig. 1 Firstly, analyzing the domains of the ontology: attempt to establish the ontology database of Dongxiang ethnic domains; secondly, specify the purpose of establishing the ontology to form hierarchical knowledge system to provide the underlying data and resources for the subsequent application; thirdly, specify the target users of the ontology: one group of users are the public, teachers and students, another group of users are developers who are the experts of the field, the developers and maintainers of the ontology. This research adopts OWL (Web Ontology Language) of W3C (World Wide Web Consortium) as the description language and Protégé 5.0 as the development tool.

Achieve the Concepts of Dongxiang Ethnic Education Resources. This research divides knowledge into declarative knowledge of facts and procedural knowledge of how to accomplish cognitive activities in the light of ACT-R theory [5]. In the domain of Dongxiang ethnic group, religion, population and enclaves etc. belongs to declarative knowledge; Huaer (Dongxiang ethnic songs) belongs to procedural knowledge, and it needs to master the basic tunes, and then put the lyrics together according to tunes and put into personal feeling. These two kinds of knowledge are related. Declarative knowledge is the foundation, and the prior procedural knowledge helps to learn new procedural knowledge.

After specify the purpose and domain of the ontology, it is necessary to extract the key vocabulary. The vocabulary is the terminology of knowledge representation in Dongxiang ethnic knowledge ontology, including conceptual class, attribute class and instances. We can get vocabulary with the help of references. The vocabulary can be divided into three parts. The first part is about the basic vocabulary, such as the ethnic overview, history, population and distribution. The second part is about the material 
culture, such as handcraft works, architecture and foods. The third part is about spiritual culture, such as religion, language, words, arts, literature, fine arts and music, folk songs, Hezhou Huaer, etc. If there exists semantic overlaps and undetermined attributes.

The Hierarchical Concepts Structure of Dongxiang Ethnic Education Resources. In its broad sense, Ontology concept is other a concept on common, or the task, function, behavior and reasoning process etc. These concepts usually form a classification hierarchy. High level classes represent the most abstract physical concepts. Because the class is inherited, the subclass inherits the abstract features of its super class. The inheritance of knowledge can make the knowledge database less redundancy, and can be reused and shared. All of the individuals in the OWL are members of the class OWL: Thing, the user-defined class is a subclass of OWL: Thing. For example, Dongxiang ethnic group is the subclass of Chinese nationalities, and it will inherit the attributes and rules of Chinese nationalities.

The selection of classes in the research is based on the consideration of three aspects. The first one is the basic requirement. The second the requirement of learning theory. The third one is the requirement of authority. We divided the knowledge in Dongxiang ethnic domain referring to the book "The inheritance of ethnic culture and the curriculum reform of ethnic basic education" which divided ethnic culture into material culture, spiritual culture, and institutions and customs. Thus there are 4 top-level conceptual classes, 15 second-level conceptual classes, 12 third-level conceptual classes, and dozens of forth-level conceptual classes. These conceptual classes can fully cover all the knowledge points of Dongxiang ethnic group. According to the basic principle of class hierarchy, form a tree structure of the ontology classes for the knowledge points of Dongxiang ethnic group, showing as Fig. 2.

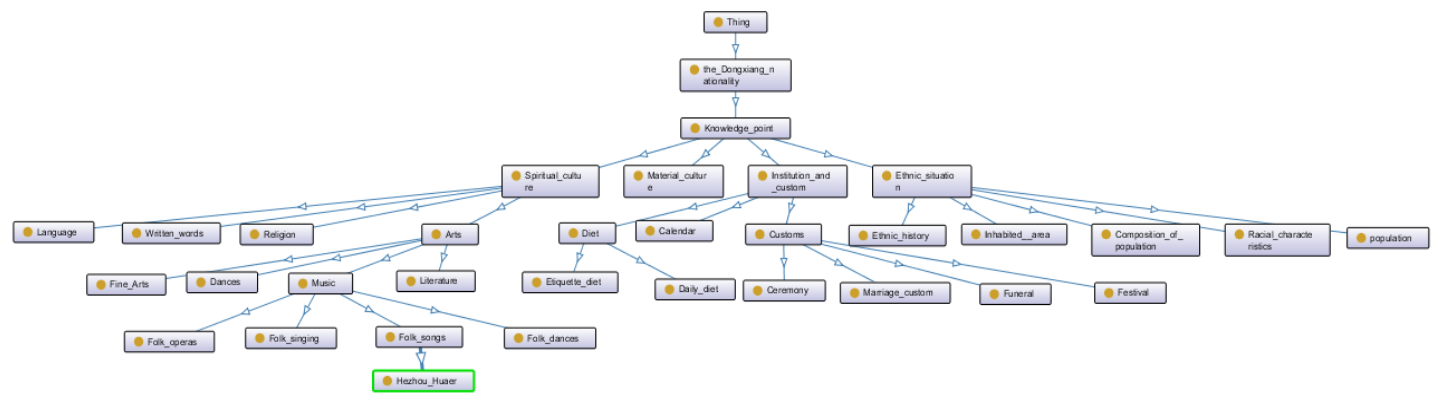

Figure 2. The ontology classes and the hierarchy of the knowledge points

The Conceptual Attributes and Relations of Dongxiang Ethnic Education Resources. Several problems should be solved before determine the dominical attributes and relations of Dongxiang ethnic group. Firstly, the common attributes of the knowledge points should be determined. These common attributes include general attributes and teaching attributes. Secondly, the special attributes of different knowledge points should be determined. Thirdly, it is how to classify and manage attributes effectively. In order to classify and manage attributes, the research employs slot[6] to describe the attributes of knowledge points ontologies and learning resources ontology. There are three kinds of slots: attribute slots, corresponding to data attributes, usually are nouns; relation slots, corresponding to objects attributes, usually are verbs; constraint slots, to limit attribute slot and relation slot, such as the types of slot values, restricted ranges of values, relation constraints, applications of axioms.

According to the above theory, the research divided the attribute slots of knowledge points ontologies into common attributes and special attributes. Common attributes include general attributes and teaching attributes. General attributes are all knowledge points ontologies should be added. Teaching attributes can assist students' learning. Special attributes are set in order to be convenient for extracting attributes of different knowledge points, and to describe the contents of knowledge points from the perspective of 
semantics. Because of the extraction of special attributes depend on specific knowledge points, Dongxiang music will be illustrated in next part. The special attributes of Dongxiang music can be described from four aspects, which are types (including folk music, literati music, religious music and palace music), ethnic group, music instruments, and spreading (including areas, periods, figures, and forms)[7].

Knowledge points are associated with each other. The completion of knowledge point ontology needs to set relation slot to form a complete system of knowledge. And the description of inner relation can meet meaning construction of constructivism and hierarchical network model of information processing theory. According to the characteristics of Dongxiang ethnic education resources database, firstly relation slot can be defined as proposed precursor relation, proposed subsequent relation and interrelation. Then the relation between knowledge points should be defined. In view of knowledge points in the domain of Dongxiang ethnic group are different from subject knowledge which have strong logical link, therefore, these knowledge points can be defined simply as father-child relation and brotherhood relation.

The Ontology Instance of Dongxiang Ethnic Education Resources. The final step of constructing ontology framework is create a instance with acquired knowledge class to realize the assignment and definition of attribute slot, relation slot and constrained slot. There are three methods to create ontology instance [8]. The first one is to create via Individuals interface of Protégé. The second one is to create via batch import of system, mainly import the processed Excel sheet of instance data into ontology database by means of API of Jena. The third one is to create ontology instance via system, that is to create instance through API programming of Jena.

The research creates instance with the first method. Fig. 3 is the illustration of the instance creation of Hezhou Huaer.

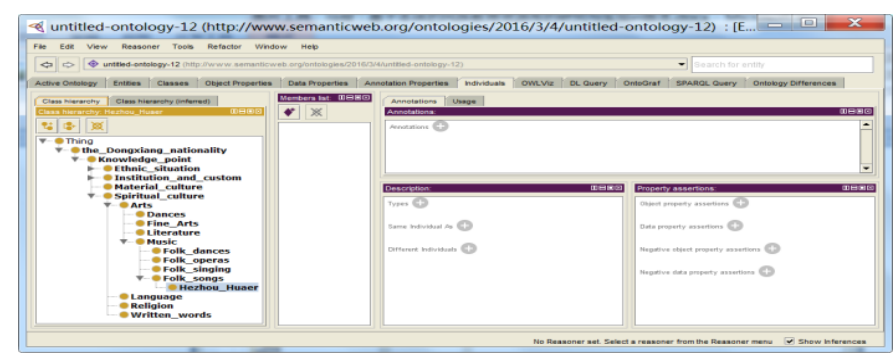

Figure 3. The ontology instance of Hezhou Huaer

\section{Conclusions}

Ontology construction is an advanced method. protégé is also an excellent platform. Both of them can improve the construction of ethnic education recourses database. They can make the construction of ethnic education resources database simple, convenient and operational.

\section{Acknowledgement}

This research was financially supported by the Planning Fund Program of Philosophy and Social Science in Gansu (No:YB017).

\section{References}

[1] Gruber T R.A Translation Approach to Portable Ontology Specifications [J]. Knowledge Acquisition, 5 (1993)199-220. 
[2] Studer R, Benjamins V R, Fensel D. Knowledge Engineering Principles and Methods [J].Data and Knowledge Engineering,,25(1998)161-197.

[3] Wang China, H Chen Wei. Teaching-Material Design Center: An ontology-based system for customizing reusable e-materials [J].Computers and Education,, 46(2006)458-470.

[4] Zhu Zhiting, et al. New Trend and Dynamic Mechanism of Digital Educational Resources [J]. China Educational Technology, 2 (2012)1-5.

[5] Anderson J R.ACT: A Simple Theory of Complex Cognition [J]. American Psychologist, 51(1996)355-365.

[6] Zhang Shouxue, Yan Bo, Gao Dongjie. Construction of Knowledge Base Based on Ontology [J]. Computer Era, 4(2014)45-46.

[7] Li Tiancheng. Construting an Ontology for Chinese Fold Music [D]. Shanghai: Shanghai Jiao Tong University, 2014.

[8] Xu Ruzhi, Yang Feng. Knowledge Sharing of Virtual Organization Based on Ontology [M]. Beijing: China Financial \& Economic Publishing House, 2010.

[9] Yuan Hua. Intellectual property protection in the construction of Digital Information Resources. Journal of Shanghai Jiao Tong University, (2003).

[10] Tang Zhenghua. Study on the principle of building college self built database. Library work study, 19 (2009). 